\title{
LASER DOPPLER VIBROMETER BASED EXAMINATION OF THE EFFICIENCY OF INTRODUCING ARTIFICIAL DELAMINATIONS INTO COMPOSITE SHELLS
}

Kamila Kustroń, Ph. D.

Institute of Aeronautics and Applied Mechanics, Poland

\begin{abstract}
During its operation, the laminate shell of the watercraft hull can be exposed to local stability losses caused by the appearance and development of delaminations. The sources of these delaminations are discontinuities, created both in the production process and as a result of bumps of foreign bodies into the hull in operation. In the environment of fatigue loads acting on the hull, the delaminations propagate and lead to the loss of load capacity of the hull structure. There is a need to improve diagnostic systems used in Structural Health Monitoring (SHM) of laminate hull elements to detect and monitor the development of the delaminations. Effective diagnostic systems used for delamination assessment base on expert systems. Along with other tools, the expert diagnostic advisory systems make use of the non-destructive examination method which consists in generating elastic waves in the hull shell structure and observing their changes by comparing the recorded signal with damage patterns collected in the expert system database. This system requires introducing certain patterns to its knowledge base, based on the results of experimental examinations performed on specimens with implemented artificial delaminations. The article presents the results of the examination oriented on assessing the delaminations artificially generated in the structure of glass- and carbon-epoxy laminates by introducing local non-adhesive layers with the aid of thin polyethylene film, teflon insert, or thin layer of polyvinyl alcohol. The efficiency of each method was assessed using laser vibrometry. The effect of the depth of delamination position in the laminate on the efficiency of the applied method is documented as well.
\end{abstract}

Keywords: watercraft hull, laminate, artificial delamination, SHM diagnostics, advisory expert system knowledge base

\section{INTRODUCTION}

The durability of the watercraft hull structure affects considerably the voyage safety and is subject to demanding requirements from maritime surveillance institutions.

The developed hull structures are oriented on the use of intelligent materials and structures. Research activities in this area are directed towards gradient and self-repairing materials, new technologies of production of elements and combining them into modules, subassemblies and the final structure, new solutions and methods of system operation administration, new and/or modernised methods to analyse correlations of links/factors in the human being/technology/ environment (C-T-O) macrosystem, new applications of mechanics methods and related techniques, modelling methods to simulate operating processes, and/or methods of structure diagnostics to assess its technical state.

The present approach to structural durability of watercraft hulls defines the durability as structure's robustness to the agents which lead to the degradation of material properties or structural integrity of the hull operating in the corrosion/ fatigue environment. In normative terms, the need to preserve sufficient watercraft hull durability imposes certain conditions to ensure material consistency and integrity of structural joints of the hull at the macroscopic level in real operating conditions. The loss of consistency or integrity is caused by damage development to the scale in which part of the construction material which is free from damages, or a weakened joint, is not able any longer to carry the operating load. The damages can be initiated at different levels of matter organization: as (1) the development of discontinuities formed during production processes of materials, elements, subsystems, and their assembly into a final product, or (2) the development of operating damages caused by local transgression of permissible loads due to bumps of foreign bodies. The further course of the discontinuity development depends on the type of construction material, the applied design solutions, and/or other agents acting on the hull structure in the $\mathrm{C}-\mathrm{T}-\mathrm{O}$ macrosystem during the hull operation.

The watercraft hull durability created at the preoperational stage should ensure macroscopic consistency of its structure by preserving a safe level of stresses and deformations generated by external and internal loads acting on the hull during its 
operation. Moreover, possible damages should develop only locally within the range which is acceptable from the point of view of safety requirements, and should be able to be detected and monitored using non-destructive methods. The durability is to be ensured by an effective diagnostic system which will detect and assess the degradation or destruction processes.

Modern engineering structures are developed with orientation towards intelligent applications [4]. One of solutions here is intelligent diagnostics. Applying a system of integrated diagnostics based on intelligent solutions enables to obtain a more robust and trustworthy structure, as a result of real assessment of its technical state $[5,6]$.

Two philosophies are applied when designing ship hulls. One of them consists in designing the hull structure in accordance with the applicable regulations, with further verification, based on real and virtual experiments, that in the assumed lifetime of operation the integrity of joints and material consistency will be preserved at the assumed safety level. The other philosophy takes into account the fact that all materials contain internal discontinuities resulting from technological processes, and these discontinuities can develop during the hull operation only up to a level at which the fatigue crack type damage does not jeopardise the metal structure of the ship hull and therefore can be permitted. Dural structures of ship hulls should ensure their safe use by revealing sufficiently high resistance to fatigue, corrosion, and external damages. When well recognised in experimental examination, the fatigue processes of dural materials used in ship hull building make the basis for preparing structural inspection procedures. Dural materials are constantly improved with respect to their fatigue crack resistance. Designs of modern hulls make frequent use of laminates due to their high fatigue and corrosion resistance, and the progressive increase of their use is observed $[10,16]$.

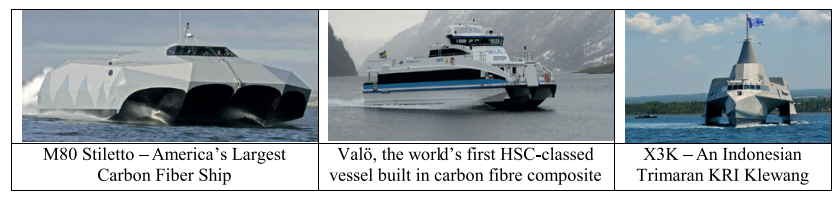

Fig. 1. Laminate structures of contemporary watercraft units [15]

Laminates reveal high corrosion and fatigue resistance, but when they are exposed to bumps of foreign bodies they crack. Damages resulting from low-energy bumps, especially those which cannot be observed from outside, can lead to internal integrity loss between composite layers, a process which is recognised as delamination. This type of damage is dangerous for laminates and should be detected in the early phase of existence, as its propagation in the fatigue load environment poses a threat to the safety of the structure.

During its operation, the thin-walled structure of the composite watercraft hull shell is susceptible to local stability losses resulting from the appearance and development of delaminations. These delaminations appear as a consequence of the development of material discontinuities generated during the lamination process, when compression stresses act on the laminate. They can also be initiated during the hull operation, when the laminate hull shell is exposed to bumps of foreign bodies. Especially dangerous are low-energy bumps, as they do not cause macroscopic damages which can be observed from the outside, and lead only to local loss of adhesive connection between the laminate layers. The appearance and development of these discontinuities can remain undetected. When propagating, the discontinuities initiate delaminations in the layered composite, which poses a threat of stability loss of the composite shell exposed to operating fatigue loads, especially compression loads. Therefore there is a need to improve tools used to diagnose the technical state of the ship hull for the appearance and development of delaminations [1-3, 9, 11-13]. Among other methods, elastic Lambda waves generated in thin-walled structures can be used for this purpose $[8,14]$.

When designing the ship hull, certain preventive measures should be named which will protect the hull against damages, including delaminations. The effect of damages on ship's safety is assessed based on observations of other real structures and modelling the observed behaviours in experiments with the newly designed structure. The time of experiments performed during the design stage should be shortened by performing intensive tests. Their only goal is to shorten the time of experiment, and it needs to be examined whether the speeding-up procedures do not affect the structure damage criterion, whether the damage mode (type) is preserved, and whether other modes which would change the nature of damage propagation and structure destruction are absent. Theoretically, the accelerated tests, in which additional modes associated with acceleration are observed, can also be performed. However, for the accelerated tests, the behaviours which are characteristic for the observed mode and for the associated modes should be extracted, which is a difficult process.

Observing the behaviour of a structure exposed to simulated operating loads enables to assess the range of its safe operation. An aspect of highest importance here is to attribute certain symptoms of gradual loss of material consistency and/or integrity of structural joints to successive phases of the experiment. These symptoms are used in diagnostics. The recognised symptoms make a basis for assessing the remaining time of operation defined by the residual durability. Particular phases depend on the adopted time intervals, referred to as observation windows. The lower limit for the adopted time interval is the sampling interval, while the upper limit can be defined by the linearity, or quasi-linearity, of the behaviour of the symptom. The observation of the structure in operation should be oriented on detecting the abovementioned symptoms. This problem can be dealt with in two ways: (1) based on experimental tests the times of successive inspections are determined during which the technical state of the structure is examined using non-destructive tests - NDT, or (2) passive and/or active sensors, introduced to the structure, make it possible to detect the symptoms online during structure operation. The latter approach is more effective, both economically and in safety terms, as it eliminates the problem of access to diagnosing 
points, and reduces, or totally eliminates, the effect of human interference. Moreover, it has a wider applicability scope and increases the technical readiness, thus taking care of the quality of service offered for the final user. It is noteworthy, however, that this higher efficiency bases on the following assumptions: (1) the system of sensors has been selected correctly and optimally for the given structure, and (2) its reliability is high and does not affect the reliability of the structure, in particular it does not generate false alarms.

The efficiency of the diagnostic systems used for delamination assessment is improved by implementation of expert systems. The diagnostic advisory expert systems make use of, among other tools, non-destructive test methods which consist in generating elastic waves in the examined structure and comparing the recorded signal with the damage reference pattern. This system requires introduction of reference patterns to the knowledge base of the expert system. The knowledge base is obtained from experimental examinations performed on specimens with implemented artificial delaminations.

The article presents the evaluation of efficiency of diagnostic methods which introduce artificial delaminations into composite shells made of glass- and carbon-epoxy laminates in order to define the reference delamination patterns for the expert system. The performed tests made use of three methods to introduce artificial delamination to the structure, and the modern diagnostic method based on the laser Doppler vibrometer to detect it.

\section{CHARACTERISTICS OF SPECIMENS USED IN TESTS}

The article reports results of tests oriented on evaluating the delamination area and position depth in specimens with delaminations artificially generated between the composite layers. The specimens, prepared from glass- and carbonepoxy laminates, had the A5 size. The generated artificial delaminations had the form of non-adhesive areas created between the laminate layers during the composite production process. These areas were created using three methods, by introducing: (1) a thin polyethylene film, (2) a teflon insert, and (3) a thin layer of polyvinyl alcohol. The delamination areas had the shapes of isosceles trapeziums with bases of 5 and $20 \mathrm{~mm}$ in length, arranged in such a way that the longer base is situated alternately at the left and right side of the specimen (Fig. 1a).

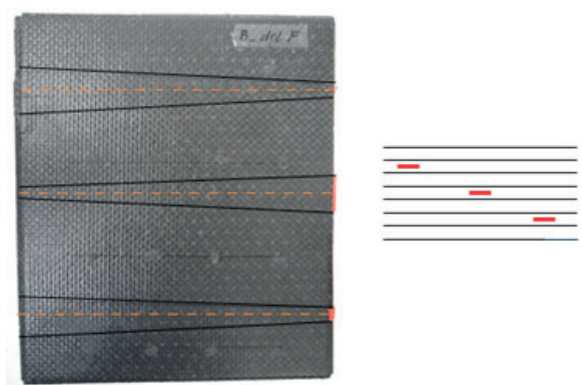

Fig. 1. A5 specimen made of carbon-epoxy laminate with local separation layers alternately introduced to obtain delaminations having the assumed areas and the shapes of isosceles trapeziums with bases of 5 and $20 \mathrm{~mm}$
The above structure of the specimen was selected to obtain the information for the knowledge basis of the advisory expert system, which was the main goal of the research reported in the article. These specimens will also be used in further tests, and the adopted system should facilitate evaluation of the measurement uncertainty at the next stage of construction of the diagnostic system based on piezoelectric sensors permanently integrated with the ship hull structure.

\section{METHODOLOGY OF EXAMINATION}

The tests were performed in the Engineering Institute, Los Alamos National Laboratory, USA, using the measuring set schematically shown in Fig. 2.

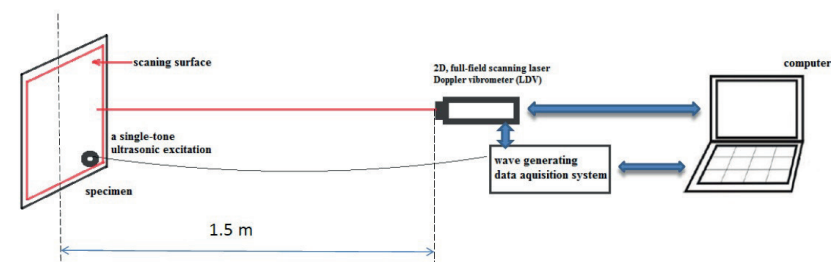

Fig. 2. Scheme of the test rig used for evaluation of the area of artificial delamination in the laminate structure

The examined specimen was mounted vertically at the distance of $1,5 \mathrm{~m}$ from the laser Doppler vibrometer. A high-frequency piezo activator which generates constantfrequency waves was wax-glued on the specimen. The assumed vibration frequency was equal to $80 \mathrm{kHz}$, following expert recommendations by Erick Flyn of Los Alamos National Laboratory. During the test, Lambda waves were generated in the specimen and, simultaneously, the surface of the specimen was scanned using the 2D Doppler laser. The visualisation was obtained via automatic estimation based on the assessment of the wave number, pixel-by-pixel, being the response to the excitation in the wave number domain [7]. The advantage of the applied method is that it does need reference patterns. Anomalies in the image indicate the presence of deformations, which are larger in places with delaminations due to lower stiffness of these areas caused by the loss of interlayer integrity.

The obtained results are presented in $3 \mathrm{D}$ using an additional map of colours. These $3 \mathrm{D}$ images can be used to evaluate the depth of delamination, measured from the surface of scanning.

The specimens prepared for the tests are collated in Table 1, which in successive columns shows the number of the specimen, its label, and basic data concerning the reinforcement material (glass, carbon) and type of material used to generate local delamination (teflon, polyvinyl alcohol, polyethylene film). 
Table 1. Specimens prepared for testing the efficiency of introduction of artificial

\begin{tabular}{|c|l|c|c|c|c|c|}
\hline $\begin{array}{c}\text { No of } \\
\text { speci } \\
\text { men }\end{array}$ & $\begin{array}{c}\text { Symbol } \\
\text { of } \\
\text { specimen }\end{array}$ & $\begin{array}{c}\text { Glass fabric } \\
\text { reinforceme } \\
\text { nt }\end{array}$ & $\begin{array}{c}\text { Carbon fabric } \\
\text { reinforcement }\end{array}$ & $\begin{array}{c}\text { Teflon } \\
\text { T }\end{array}$ & $\begin{array}{c}\text { Polyvinyl } \\
\text { alcohol } \\
\text { A }\end{array}$ & $\begin{array}{c}\text { Film } \\
\text { F }\end{array}$ \\
\hline 1 & A_delA & + & - & - & + & - \\
\hline 2 & A_delF & + & - & - & - & + \\
\hline 3 & A_delT & + & - & + & - & - \\
\hline 4 & B & - & + & - & - & - \\
\hline 5 & B_delA & - & + & - & + & - \\
\hline 6 & B_delF & - & + & - & - & + \\
\hline 7 & B_delT & - & + & + & - & - \\
\hline
\end{tabular}

Six specimens shown in Fig 3 were used in the tests.

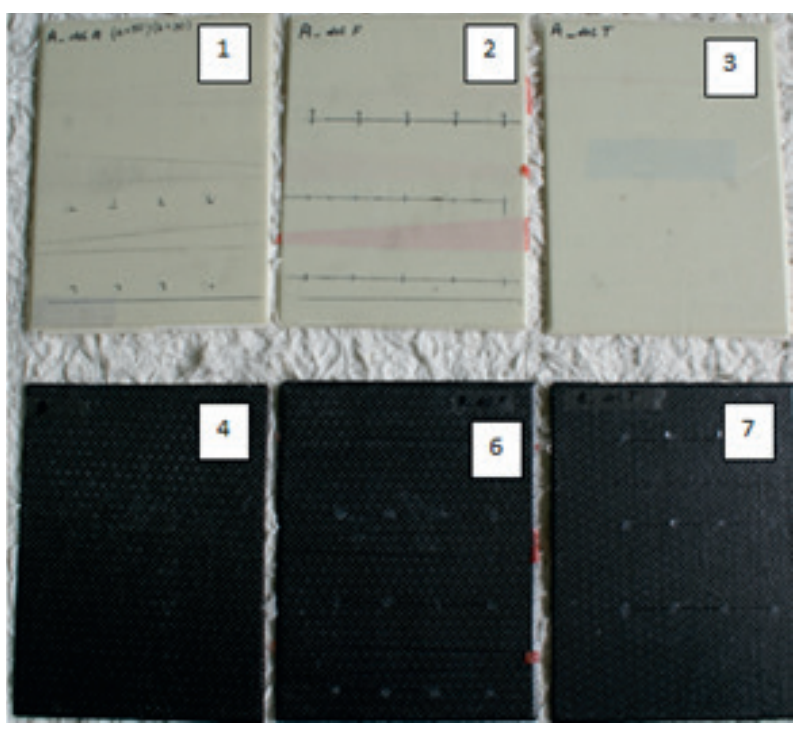

Fig. 3. Photo of the set of specimens used in the examination performed with the aid of the laser Doppler vibrometer to assess the areas of delamination

Specimens 1 to 3 were prepared from glass-epoxy laminate. During the delamination introduction process, all trapezoidal artificial delamination areas of the specimens were filled with: (1) polyvinyl alcohol with a thin copper wire, or (2) thin polyethylene film, or (3) rectangular teflon insert. Specimens 4 to 7 were prepared from carbon-epoxy laminate. Specimen 4 was pure, without artificial delamination, while specimens 5 to 7 had delamination areas filled with polyvinyl alcohol, thin polyethylene film, and rectangular teflon insert, respectively, like in case of the glass-epoxy specimens.

Specimen 5 made of carbon-epoxy laminate with local artificial delaminations introduced using polyvinyl alcohol was damaged during annealing in the production process.

\section{RESULTS OF TESTS}

The analysis of the test results having the form of the recorded images clearly detects the delaminations. The method of their detection is very effective, fast, and efficient. The recorded images have made a basis for evaluating the efficiency of particular delamination introduction methods. Covering Specimen 1, made of glass-epoxy laminate, (Fig. 4), with polyvinyl alcohol did not result in local adhesion loss (Fig. 5), and the only visualised damage was caused by removing the specimen form the mould.
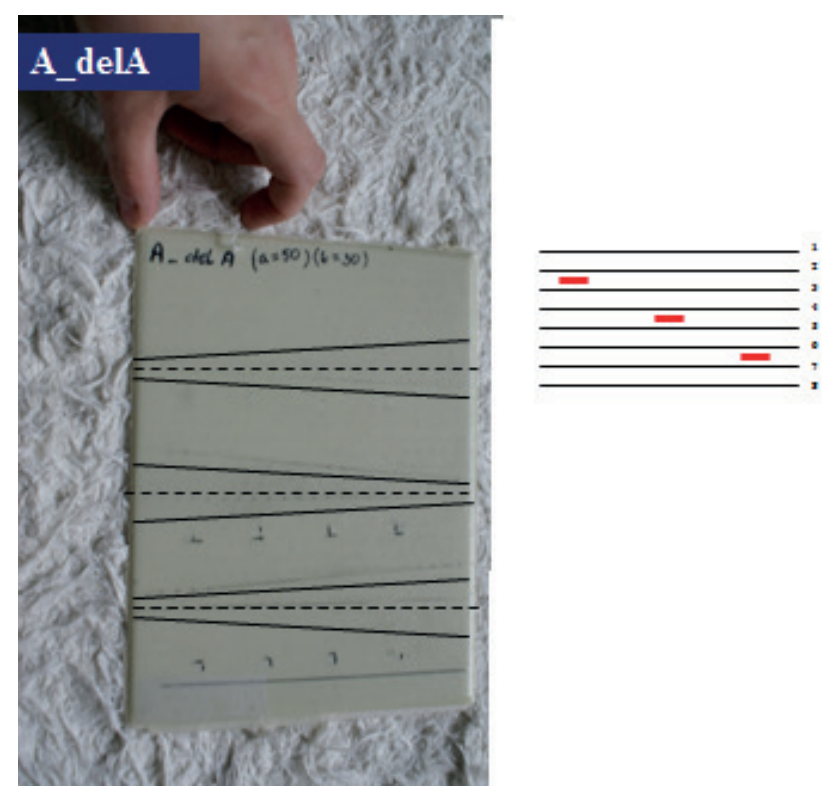

Fig. 4. View of Specimen 1, made of glass-epoxy laminate. To generate delamination, the places marked as trapeziums were covered with polyvinyl alcohol between layers 2 and 3, 4 and 5, and 6 and 7 in the production process

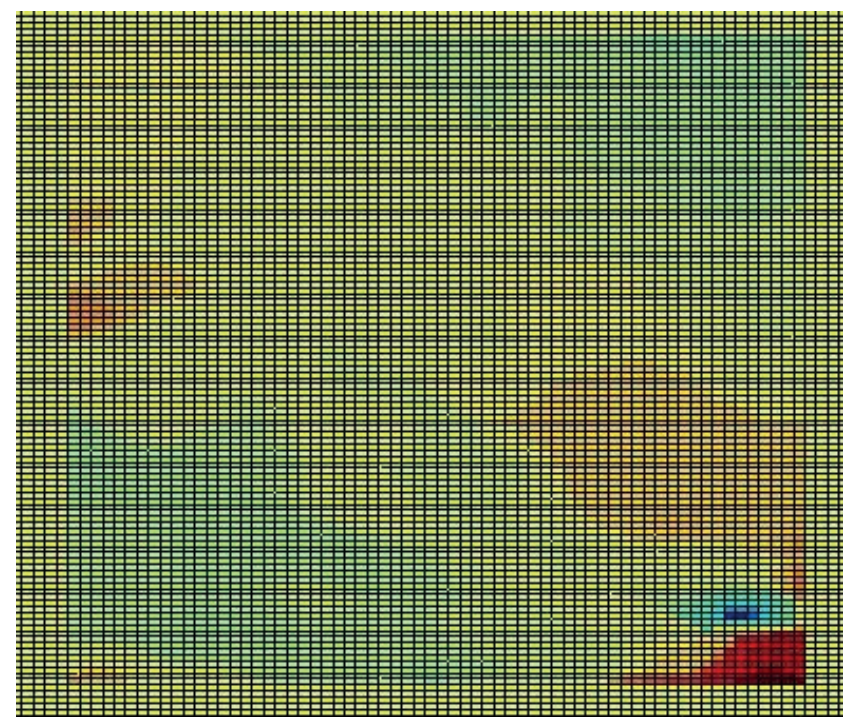

Fig. 5. Visualisation of Specimen 1, made of glass-epoxy laminate with trapeziums covered with polyvinyl alcohol to provoke local adhesion loss and further delamination between layers 2 and 3, 4 and 5, and 6 and 7

During the production process, a copper wire was introduced to the places covered with polyvinyl alcohol to enable the generation of additional high-voltage discharge, and thus provoke the appearance of delamination being the object of further examination.

Specimen 5 made of carbon-epoxy laminate with trapeziums covered with polyvinyl alcohol was damaged during annealing.

Three thin trapezium shaped films were introduced to Specimen 2, made of glass-epoxy laminate, between layers 2 and 3, 4 and 5, and 6 and 7 (Fig. 6). 


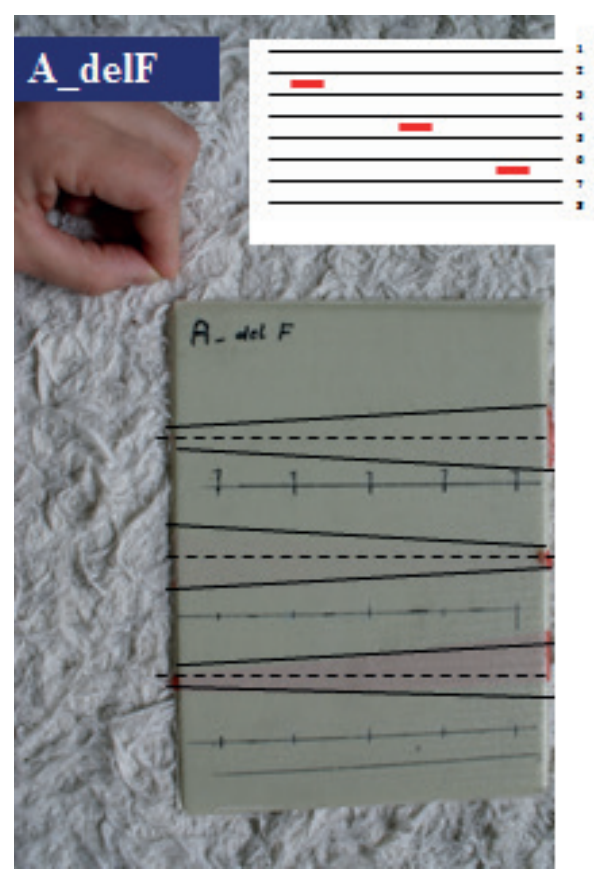

Fig. 6. View of Specimen 2, made of glass-epoxy laminate with places of introduction of trapezium shaped polyethylene films between layers 2 and 3, 4 and 5 , and 6 and 7

Figure 7 shows visualisation of elamination of Specimen 2.
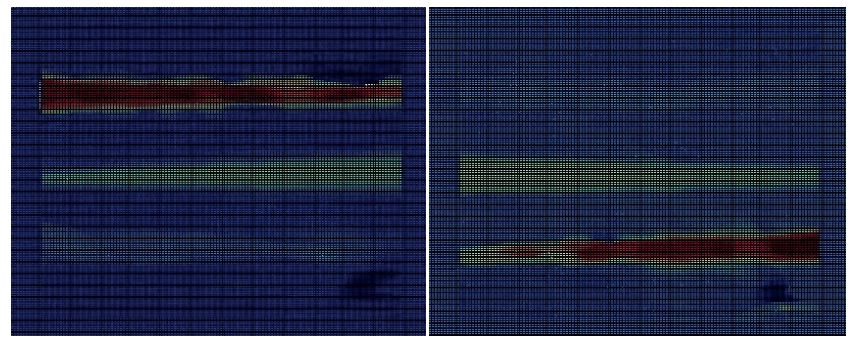

Fig. 7. Visualisation of delamination of Specimen 2. Internal delamination obtained when the trasducer was glued at the bottom left corner on the smooth side of the specimen and the rough surface was scanned (left), and when the trasducer was glued at the bottom left corner on the rough side of the specimen and the smooth surface was scanned (right)

On the left-hand side, Fig. 7 shows the visualisation of internal delaminations obtained by exciting the Lamba waves with the aid of the trasducer glued at the bottom left corner on the smooth surface of the specimen and scanning the rough surface, while the visualisation shown on the right-hand side was obtained in the opposite situation, i.e. when the trasducer was moved to the bottom left corner of the rough surface and the smooth surface of the specimen was scanned.

Figure 7 shows clear patterns of internal delaminations, the used colour palette illustrates the depth of delamination position. The patterns on the left-hand and right-hand sides are similar to each other. The right-hand pattern can be obtained after transforming the left-hand pattern by rotation by 1800 with respect to the bottom right corner, which reflects the arrangement of the delaminations purposely introduced to the structure of the laminate. The performed examination indicates that the efficiency of generating artificial internal delaminations by introducing the films during the production process is high; the images of the introduced films are almost identical in both cases. We can also conclude that the side on which the trasducer is glued and the side along which the specimen is scanned do not affect the results of the examination. Further research is to be performed to separate the trasducer position/surface smoothness relation, from that between the surface smoothness and the scanned pattern.

The method to generate delamination by inserting a thin polyethylene film has also turned out effective for carbonepoxy laminates.

However, unlike the test with the glass-epoxy laminate, here scanning Specimen 6 (Fig. 8), made of carbon-epoxy laminate with thin film introduced to generate delamination, has revealed some effect of trasducer position and scanned surface smoothness on the recorded results. More precise evaluation of this effect requires additional tests. The visualisation patterns on the left-hand and right-hand sides clearly differ between each other, by colours attributed to particular delaminations and depths of their positions in the laminate, rather than by delamination shapes themselves.

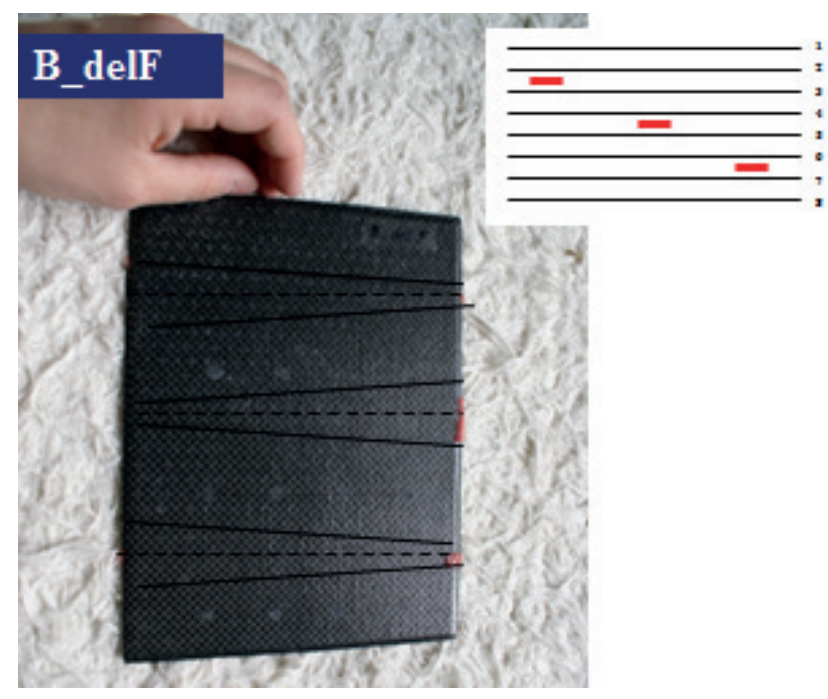

Fig. 8. Carbon-epoxy laminate with

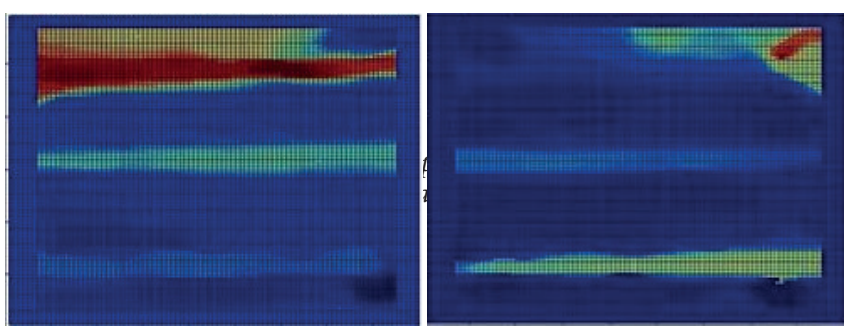

Fig. 9. Visualisation of delamination of Specimen 6 made of carbon-epoxy laminate with artificial delamination generated by introducing a thin film. The trasducer was glued on the smooth side of the specimen and the rough side was scanned (left), and trasducer was glued on the rough side of the specimen and the smooth side was scanned (right).

For Specimen 7 (Fig. 3 and Table 1), made of carbon-epoxy laminate, a rectangular teflon insert with a copper wire was introduced into it, and separately a cooper wire alone, see Fig. 10. If no delamination had been recorded, the specimen was planned to be exposed to high-voltage discharge. 


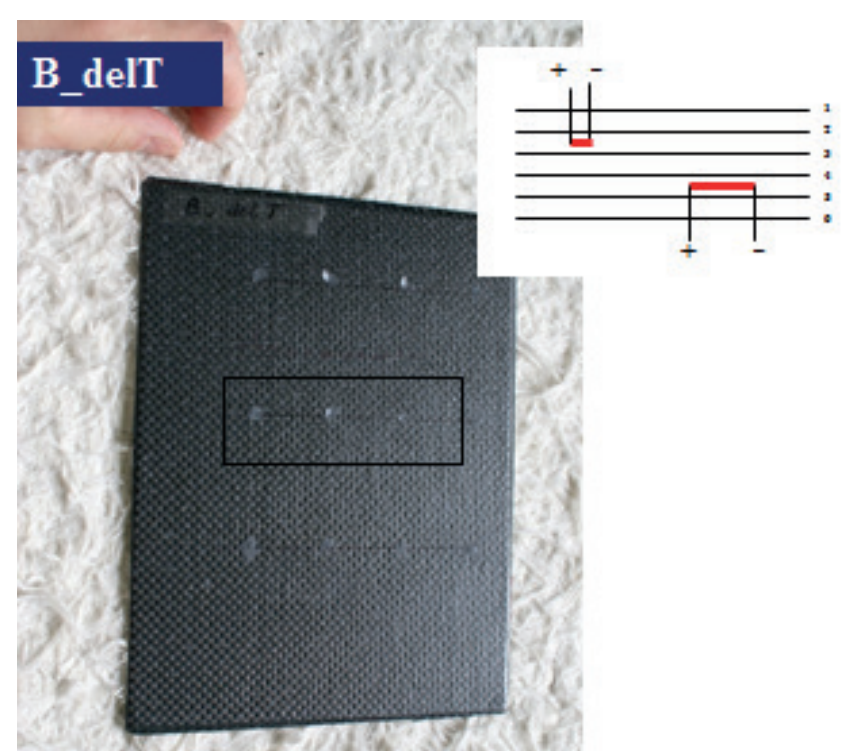

Fig. 10. Specimen 7, made of carbon-epoxy laminate, with (1) rectangular teflon insert and copper wire, and (2) a separate copper wire introduced during the production process

The visualisation did not detect the presence of delamination (Fig. 11). Here, the introduction of teflon turned out ineffective, as far as the goals of the reported research were concerned.

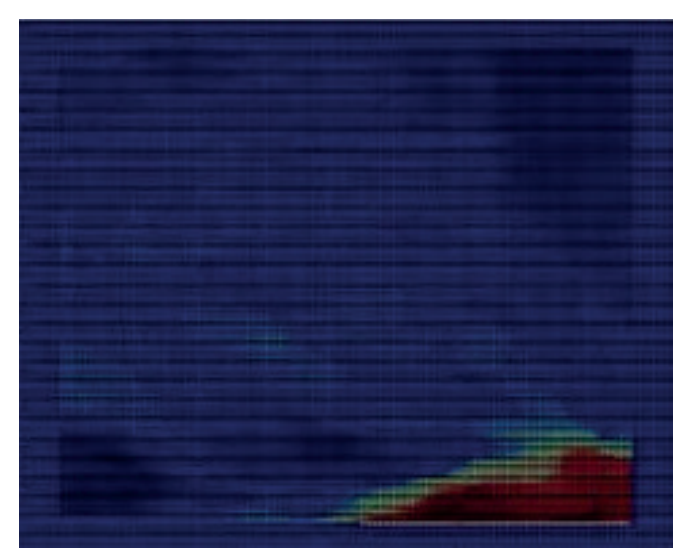

Fig. 11. Visualisation of Specimen 7 made of carbon-epoxy laminate, with locally introduced teflon insert. The trasducer was glued on the smooth surface of the specimen and the rough surface was scanned

\section{CONCLUSIONS AND DIRECTIONS OF FURTHER RESEARCH}

The article presents the results of the research oriented on evaluating the efficiency of different methods to introduce delamination into structures of glass- and carbon-epoxy laminates. The delamination was obtained by generating, in the production process, local non-adhesive areas between the laminate layers. A thin layer of polyvinyl alcohol, a thin polyvinyl film, and teflon were used for this purpose. The efficiency of particular methods was evaluated using the Laser Doppler Vibrometer. The highest efficiency was obtained using the thin polyethylene film, which was confirmed by the recorded visualisation images. Further research should analyse in more detail the effect of surface smoothness on the introduction of Lambda waves and on the results of scanning.

The performed tests have also proved the efficiency of the applied method in assessing the depth in which the delamination takes place, i.e. between which layers. The efficiency of this method decreases with the increasing depth of delamination. The laminate thickness limits for which the method can be applied should be assessed taking into account the type of reinforcement fabric, its thickness, and volumetric percentage of the reinforcement and the matrix (binder) in the laminate.

The research was performed during author's stay in the Los Alamos National Laboratory, within the framework of the grant for study visit financed from the Development Programme of the Faculty of Power and Aeronautical Engineering, Warsaw University of Technology, for years 2011-2015, being part of the EU Operational Programme Human Capital.

\section{BIBLIOGRAPHY}

1. Bolotin V.V. Delaminations in Composites Structures: Its Origin, Buckling, Growth and Stability. Composites: Part B Engineering, 1996; 27(2): 129-145.

2. Bolotin V.V. Mechanics of Delaminations in Laminate Composite Structures. Mechanics of Composite Materials, 2001; 3(5/6): 367-380.

3. Clarke M.J., Pavier M.P. Experimental Techniques for the Investigation of the Effects of Impact Damage on Carbon Fiber Composites. Composite Sciences and Technology, 1995; 55: 157 - 169 .

4. Dilhac J-M. Smarter. CHIST-ERA Project Seminar, 2013.

5. Farrar C. R., Worden K. An Introduction to Structural Health Monitoring. Philosophical Transactions of the Royal Society A, 365 (LA-UR-04-8385), 2007: 303-315.

6. Farrar C. R., Worden K. Structural Health Monitoring: A Machine Learning Perspective. John Wiley \& Sons, 2012.

7. Flynn E.B., Jarmer G.S. High-Speed, Non-Contact, Baseline-Free Imaging of Hidden Defects Using Scanning Laser Measurements of Steady-State Ultrasonic Vibration. International Workshop on Structural Health Monitoring, Stanford, USA, DEStech Publications, Inc. 2013.

8. Giurgiutiu, V., (2005) Tuned Lamb Wave Excitation and Detection with Piezoelectric Wafer Active Sensor of Structural Health Monitoring. Journal of Intelligent Material Systems and Structures, 2005; 16(4): 291-305. 
9. Liu S.F., Hwang G.H. Buckling Behaviour of Composite Laminates with Multiple Delaminations under Uniaxial Compression. Composite Structures, 2001; 53:

10. Mouritz A.P., Gellert E., Burchill P., Challis K., Review of advanced composite structures for naval ships and submarines. Composite Structures. Vol. 53(1),2001, pp. $21-42$

11. Oswald A., Tafreshi T. Global Buckling Behaviour and Local Damage Propagation in Composite Plates with Embedded Delaminations. International Journal of Pressure Vessels and Piping, 2003, Vol. 80.

12. Schoeppner N.J., Pagano G.A. Delamination of Polymer Matrix Composites: Problems and Assessment. Comprehensive Composite Materials, Elsevier, 2000.

13. Soni C. D., Sahoo P. K., Srinivasan S., Santosh K. Fracture Mechanics Analysis and Strength Prediction of Carbon Fiber Composite Laminate with a Delamination. JEST-M, 2012;1(1): 8-11.

14. Wandowski T., Malinowski P., Kudela P., Ostachowicz W. Guided wave-based detection of delamination and matrix cracking in composite laminates. Proceedings of the Institution of Mechanical Engineers Part C-Journal of Mechanical Engineering Science, 2011; 225: 123-131.

15. www. $3 \mathrm{mb}$.asia/the-use-of-composite-materials-innautical-applications/(on-line 30/03/2015)

16. www.compositesworld.com/articles/the-marketsboatbuilding-and-marine-2015(on-line 30/03/2015)

\section{CONTACT WITH AUTHOR}

\author{
Kamila Kustroń
}

Institute of Aeronautics and Applied Mechanics Faculty of Power and Aeronautical Engineering Warsaw University of Technology Politechniki 1 Sq. 00-661 Warszawa Poland 